\title{
DESIGN THINKING IN ACTION: A DPR CASE STUDY TO DEVELOP A SUSTAINABLE DIGITAL SOLUTION FOR LABOR RESOURCE MANAGEMENT
}

\author{
Bochen Zhang ${ }^{1}$, Ning Dong ${ }^{2}$, and Leonardo Rischmoller ${ }^{3}$
}

\begin{abstract}
The construction industry is known for cost and time overruns as well as vulnerable to mistakes and reworks, leading to low productivity compared to other industries in the last fifty years. As of today, the development of digital technologies has transformed many other industries, from automotive to transportation and finance. However, even though new tools and technologies are emerging rapidly for the construction industry, the rate of innovation and innovation adoption is low. The traditional push approach can hardly address the varied and indeterminate sets of issues faced by each project team whose solutions might be unique and evolving. The Design Thinking approach, which resembles the pull strategy in the Lean concept, has great potential in searching for and developing sustainable and innovative solutions with the users.

In this paper, the Business Process Improvement team in DPR Construction, a national technical builder, leveraged the Design Thinking approach to develop a sustainable digital solution hand in hand with the user leveraging a Low Code Application Platform. The approach yielded a result that eased the transition period for adoption and significantly improved the efficiency of the labor resource management process.
\end{abstract}

\section{KEYWORDS}

Design Thinking, Lean Construction, process, continuous improvement, technology adoption.

\section{INTRODUCTION}

\section{TeChNOLOGY Adoption in CONSTRUCTION ORganizations}

According to the World Economic Forum, the population of the world's urban areas is increasing by 200,000 people per day, all of whom need houses, water, and utilities to support modern life (Renz and Solas, 2016). As the scale of the projects is growing fast, construction managers face a bigger challenge that involves managing many aspects at the same time, such as environmental impact, resource requirements, and economic

1 Business Analyst, DPR Construction, 1450 Veterans Boulevard, Redwood City, CA 94063, BochenZ@dpr.com, orcid.org/0000-0001-7212-5150

2 Business Process Improvement Lead, DPR Construction, 1450 Veterans Boulevard, Redwood City, CA 94063, TonyD@dpr.com, orcid.org/0000-0002-8529-804X

3 Business Analyst, DPR Construction, 1450 Veterans Boulevard, Redwood City, CA 94063, LeonardoR@dpr.com, 0000-0001-6317-2692 
concerns. Therefore, construction firms should increasingly be utilizing technologies to manage projects better (Sargent et al., 2012). However, if technology adoption and its use are poorly diffused within the organization, the benefits can be limited (Peansupap and Walker, 2005). The traditional approach to adopt a new technology starts with a group of senior managers authorizing a new tool to be used within the organization. Nevertheless, studies indicate that although the rate of information technology implementations is growing and further, the adoption rate within the organization is very slow (Acar et al., 2005). Many studies have worked out sophisticated frameworks to identify factors that counter-act the adoption rate, and they all agree that applying an appropriate implementation approach that fits the company culture and the leadership model has a significant impact on adopting a new technology successfully (Sargent et al., 2012; Gambatese and Hallowell, 2011; Peansupap and Walker, 2005).

\section{DPR as An Advanced Lean Organization to Adopt Technology With A Design ThinkING APPROACH.}

DPR Construction's story begins in July of 1990. It was founded by Doug Woods, Peter Nosler, and Ron Davidowski, ("D", "P" and "R"). With a service mindset, DPR set off to be a full-service contractor serving owners within 5 core markets. As of today, DPR employs more than 7,000 employees across its 23 offices across the US (DPR 2020).

DPR is an organization that is advanced in adopting Lean construction in support of its vision: build a truly great construction company. With the spirit of "being ever forward and harnessing continuous improvement at all levels," DPR empowers its employees to search for spaces to improve and help each of the processes the company engages in to become leaner. As a result, the decision making can come from the front-line employees who are closest to the status quo of existing conditions. The top managers can therefore spend the time to focus on other immediate decision makings. This culture created a very Lean decision-making process, enabling self-initiative, self-disciplined learning and improvements for the whole organization (Maestas and Parrish, 2014). On the other side, this culture is also making the DPR's Business Process Improvement (BPI) team realize that the traditional top-down implementation model is ineffective in DPR's case. In this paper, DPR's BPI team utilized a designing mindset to develop a mobile phone-based human-centered solution with the superintendents and the foreman on the projects to solve the labor resource management problem within a relatively short period. The paper redefines the technology adoption problem in the context of a Lean culture organization. Then, the paper highlights the journey that the BPI team went through to develop the solution. Finally, the paper discusses the lessons learned and generalize that knowledge for other Lean companies to develop sustainable solutions adopting new technologies.

\section{DESIGN THINKING}

The design process has always been a catalyst for innovation processes in product development and problem-solving (Tschimmel, 2012). The research on design thinking was originally focusing on the cognitive process of designers. As the theory develops, the term has extended into the business realm and become a hot topic that leaning towards any business and organization can be creative and innovate by learning from the designers' way of thinking and solving problems (Johansson-Sköldberg et al., 2013). As of today, Design Thinking is understood as a way of thinking, which leads to transformation, evolution, and innovation, to new forms of living and managing the business (JohanssonSköldberg et al., 2013). Researchers and practitioners not only defined the ways to think 
like a designer but also explored new models of processes and toolkits, which help to improve, accelerate, and visualize every creative process (Tschimmel, 2012). Members of IGLC community have also demonstrated the power of Design Thinking process: A previous case study from the IGLC community had demonstrated leveraging Design Thinking process in developing innovative tools to help manage human processes in an innovative way (Spitler and Talbot, 2017). In another case study, the researchers had developed empathetic solutions democratically for implementing a new decision-making method throughout the organization (Schottle et al., 2019). In both cases, Design Thinking was applied to develop sustainable and innovative solutions that were well adopted by the stakeholders to solve poorly defined problems.

The Design Thinking approach comes from how the designers try to solve a design problem. Previous research (Tschimmel, 2012) has defined a couple of main characteristics of a Design Thinking approach, and here we re-organize them into four aspects:

- Design Thinking requires a deep understanding of the current condition: Intensive observation and wondering, challenging stereotypical perception.

- Design Thinking is abductive and inventive: comfortable with ambiguity and uncertain goals, challenging stereotypical perception. Emotional and rational at the same time, subjective.

- Design Thinking is visual and iterative: Mainly visual, use of sketching and prototyping tools; failure is a part of the process.

- Design Thinking is human-centered: empathic and human-driven, deep understanding of peoples' needs and dreams.

Based on these Design Thinking characteristics, several Design Thinking process models have been published and proved as effective. IDEO first proposed two of the bestknown models, such as the 3I (Inspiration, Ideation, Implementation) model and HCD (Hearing, Creating and Delivering) model. However, the 3I model does not describe the whole design process but only the idea generation phase. Although the HCD model provided a more comprehensive approach, the toolkit terms defined in the model are often considered too rigid to follow. Moreover, the Hasso-Platter-Institute's Design Thinking model emphasis on the stages of a design process are not always undertaken sequentially but may loop back to earlier phases. Furthermore, the Double Diamond model proposed the visual mapping of the divergent and convergent design thinking process. Last but not least, the Service Design Thinking (SDT) model proposed a non-linear process to design a service process with a fast iterative approach (Tschimmel, 2012). The BPI team in DPR Construction reviewed these five models and came up with a Design Thinking process to solve the labor resource management problem.

\section{CASE STUDY}

\section{The BPI and The Labor Management Problem}

In January 2019, DPR's Bay Area labor management group reached out to the Business Process Improvement (BPI) team for help to create a sustainable solution to improve their labor resource management process. The BPI team in DPR Construction is designated to look at DPR's business processes and constantly identifies opportunities for improvement by conducting interviews, analyzing data, and carrying out holistic process analysis. The 
ultimate goal for the team is to ensure continuous improvement of DPR's business operations.

DPR's Bay Area group consists of laborers ranging from 100 to more than 250 in a year. The total headcount fluctuates due to the nature of the construction market as the demands go up and down. The responsibility of this labor group is to help with miscellaneous work such as house cleaning or serve as a task team to contain any emergencies or urgent tasks. The volatile nature of this group imposes a challenge for managing the labor resource since it is hard to plan, allocate and track each of the members in the group promptly by using the managers' memories, sending emails and making phone calls. It is also risky for one manager to manage the allocation from day to day without a backup person since it is time-consuming to transfer the knowledge regarding an individual's skillsets, team dynamics, and other personal information to make appropriate staffing decisions such as sharing resources to optimize the productivity and the utilization of the labor resource. A simple centralized database storing staffing related information and tracking data seems to be an ideal solution. However, due to the current process relying on the information flying in the air (e.g., conversation, email, phone calls, and even sometimes rumors.), it is hard to collect consistent and accurate data generated through the current staffing process to feed the database.

The challenge is not new, the labor group managers had investigated software solutions in the market and reported that the existing solutions often require extra training efforts or contain features that are not needed. "We want a tool that is very simple and fits exactly our daily work, not what the others think is going to be useful for us," quote the General Superintendent of the labor group. Also, the future software users-laborers and superintendents - showed conservative attitudes toward the new technology and a very low new process adoption rate.

Before diving into the solution, the BPI team explored the current process to understand the underlying problem behind the request by conducting interviews and company-wide surveys. The general manager creates an isolated Excel sheet and sends it to the superintendents and foreman on every project for them to forecast the next month's labor requests on the project. After the project teams return their forecast, an admin person combines all the entries into one Excel table. The labor group's manager hopes that every project will forecast for their labor requests ahead of time to enable optimized planning for labor assignments. In reality, the percentage of the employees who fill out the sheet and return the sheet on time never exceeded $30 \%$. Due to the uncertainty of the tasks, the superintendents reported that the monthly forecast could hardly reflect the real need of the job site labor resources on a daily basis. Therefore there is no motivation for the superintendents to put in their forecasts since they still have to make an on-demand request to the manager to get the labor resource. In a survey, most of the project teams consider this labor forecasting process a waste of time. Moreover, the admin person deems the compilation work time-consuming and cumbersome. Through the investigation, the BPI team revealed the following critical challenges faced by the Excel-based process:

- Aging data. The superintendents and the foreman must adjust their staffing according to the project's workload, which changes dynamically due to the uncertainties on the job sites. The decisions are typically made days before the work, not months. By the time the admin compiles the Excel files, the data collected earlier in the month is outdated.

- Fragmented process. Although the superintendents and the foreman took the time to forecast, they cannon get what they need directly. Phone calls and emails are 
still mandatory for requesting labors for each project, making the forecast process a burden.

- Low mobility solution. Typically, the superintendents and the foreman spend most of their time making decisions and supervising work in the field. The current process requires them to remember their needs and update later the Excel file in the trailer office.

- Over-estimated users' technology fluency. Although filling Excel tables is easy for most of the engineers, not every superintendents and foreman are familiar with the Windows environment and can use Excel fluently.

The exploration of the current process indicates a traditional "push" approach in which managers create a tool first and push the tool to the user without full comprehension of the true needs and user conditions (e.g., skillsets or personal preferences). Such an approach can hardly gain traction in Lean driven organizations. To influence behavior through a human-centered approach, the BPI team decided to utilize a Design Thinking approach to develop a sustainable and innovative solution with the stakeholders rather than for them.

\section{Design Thinking Applied to the Labor Management Problem}

With the understanding of the labor management problem, and leveraging a design thinking approach, the BPI team proposed solution consisted of a mobile application that 1 ) is easy to use in the field without complicated training or any disruptions to the current working rhythm, 2) can automatically generate records to replace the sticky notes, paper, and isolated Excel tables for the labor managers. Instead of following the traditional "design a solution for the group," the BPI team took a non-linear problem-solving process that involved spending time observing and understanding the users' needs, interspersed with brainstorming sessions, and the iterative creation of prototypes to visualize alternative solutions addressing users' feedback. The objective was to design an application that will maximize the adoption rate to improve the group's labor resource management process through a Design Thinking approach that involves the users when designing the solution, adds value to the user's daily work and ensure a smooth transition period.

\section{The Design Thinking Process Application}

\section{Empathy: Observations and Interviews}

The BPI team started visiting project job sites in the Bay Area to understand the current condition of the resource management process. According to the initial investigation, the team identified two groups of people to observe in the field: the superintendents (labor requestor) and the labors (resource to be managed). The team follows the philosophy that to understand reality, one must merge into the project team. Therefore, the content of the site visits not only included interviews but also shadowing the users' work. Comparing to a workshop in the conference room trying to simulate the reality, the working experience created the initial empathy for the team to feel the pain of the user by actually do the work. This experience also sets up the emotional foundation for designing the solution.

Interviews were directed towards the resource management process on the project in a casual manner (i.e., let the interviewee decide where to start the interview). The conversations started with general questions about how the interviewee is currently 
managing their labor resource in the field. The BPI team then asked related questions to induce the conversation to be more detailed. Also, the BPI team avoided the conversation run into discussing "what the process should be" but focused on the status quo.

The observations and interviews yielded the following information that showed evidence of empathy built up for the current condition:

- The project needs for labor are very dynamic, and it is hard to predict labor requests for more than three weeks. There are often exempts and emergent works that require extra resources.

- Labor requests are initiated by the job site superintendents and processed by the general manager in the office. The general manager then must communicate the decision back to the superintendents and the laborers.

- Communication mainly goes through phone calls and text messages with no formal records.

- The superintendents are frustrated with the current process because it is not fully transparent, and it does not guarantee replies.

- The general manager must go through a painful process of handling daily requests and making appropriate decisions without the help of any readily data or information.

The BPI team closed the visits often by asking the interviewees to imagine a perfect world where their current complaints are solved. The conversations often rendered interesting design ideas and suggestions. The BPI team valued these ideas and suggestions as these are the requests from the users.

\section{Inspiration: Process Mapping and Brainstorm Sessions}

After the site visits, each of the three BPI team used some time to organize and absorb the information collected through observations and interviews. During this time, the team members built personal perceptions regarding the current condition of the resource management process and created sketches to map the process out. Following is a sketch note:

- Labor resource requirements are communicated on-demand by making phone calls to the general manager in the office with no records or records on paper.

- The manager put the request in a notebook and process the request leveraging information in his memory, such as project details, people's names, and background, team dynamics, and so on. Then the manager will make a resource allocation decision. Finally, the manager will send text messages to both the superintendent and the labor to confirm the allocation.

- The superintendent will confirm with the labor and share the project on-board information

- The labor will go to the project and start work on the assigned scope.

Following the personal absorbing and building of perceptions, a brainstorming session is held on the following day for all the BPI team members to build a finalized process map to visualize the current condition. Since the visual perception is dominant among the senses in communication, the BPI team mapped the process on the whiteboard (Figure 1) first to facilitate the communication within the team and to get alignments with all the team members quickly. Mapping the process on a whiteboard also helped to identify insufficient information and places for improvement easily as it enabled the team to get a 
view of the big picture quickly. The process maps are built in an iterative way involving invitations to the process stakeholders (i.e., the General Superintendent, the labor manager and the admin person) and users that validate and confirm the accuracy of the process map drafts. The BPI team conducts follow-up interviews to collect more information when some critical details are identified missing after capturing the initial information.

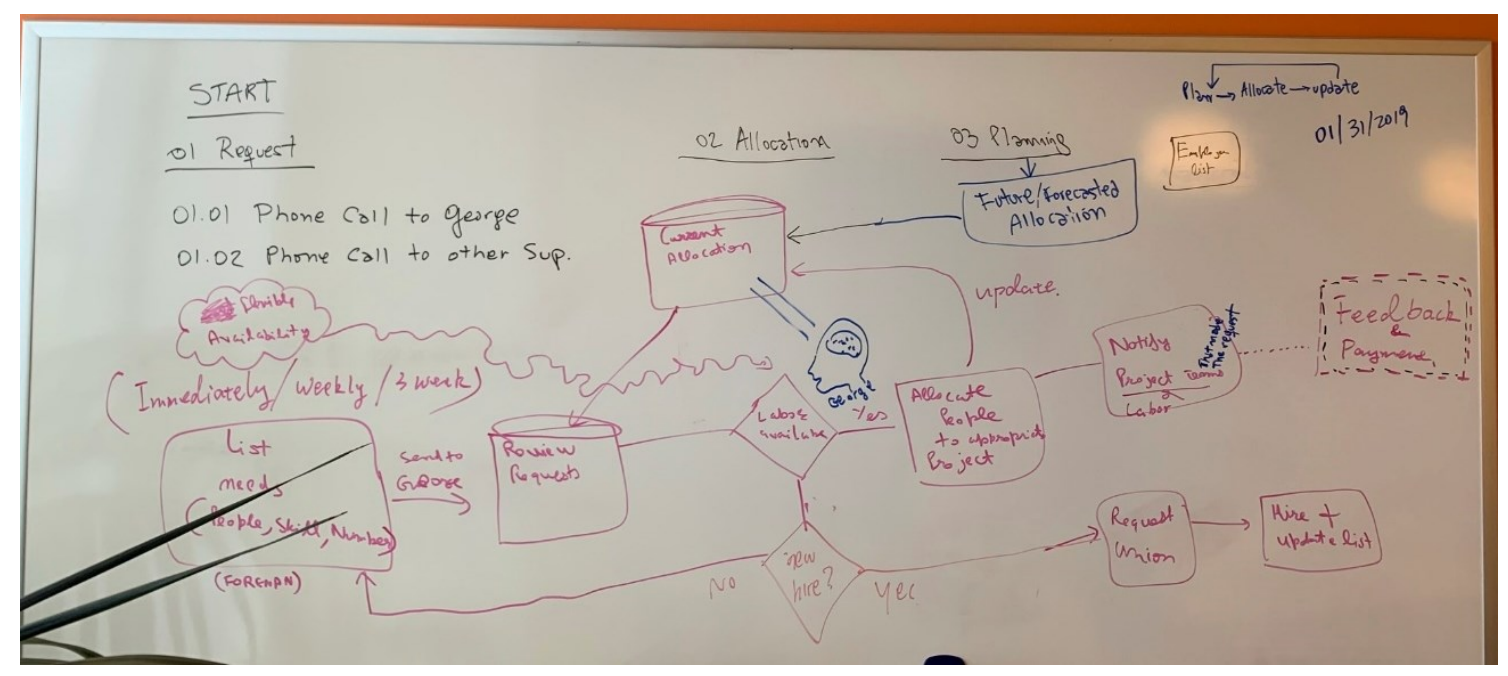

Figure 1: Process map depicting labor dispatching workflow by a superintendent on the job site created during a brainstorm session

Utilizing the process map, the BPI team identified challenges to improve in the current process as follows:

- Communication occurs through phone calls or text messages, no formal records to track or to monitor requests and allocation decisions.

- No readily data or information help the managers to make decisions, the manager must have conversations or use memories and experience to make decisions. The general manager becomes the bottleneck in the process, but no others can help.

- Sending out notifications is a manual process, which is always delayed and error prone.

By analyzing the current process, the BPI team realized that the biggest challenge is to reduce the efforts to facilitate communication and information flow between the job site and the office, amongst stakeholders and the users, including general managers, superintendents, and the workers. At the end of the brainstorm session, the BPI team decided to create a resource management platform with mobile applications build upon it to facilitate a more automatic and user-friendly resource management process for the labor group.

\section{Prototyping: Ideation Sessions and Fast Iterations}

As the BPI team identified the places for improvement, it was time to put together an action plan to tackle the problems. The BPI team held an ideation session following the brainstorm session (in this case, the two combined into one session) to design the prototype. Again, sketching on the whiteboard (Figure 2) dominates the team communication by visualizing the design concepts and ideas. These design concepts and 
ideas are tested and improved through fast prototyping and iterations with the stakeholders.

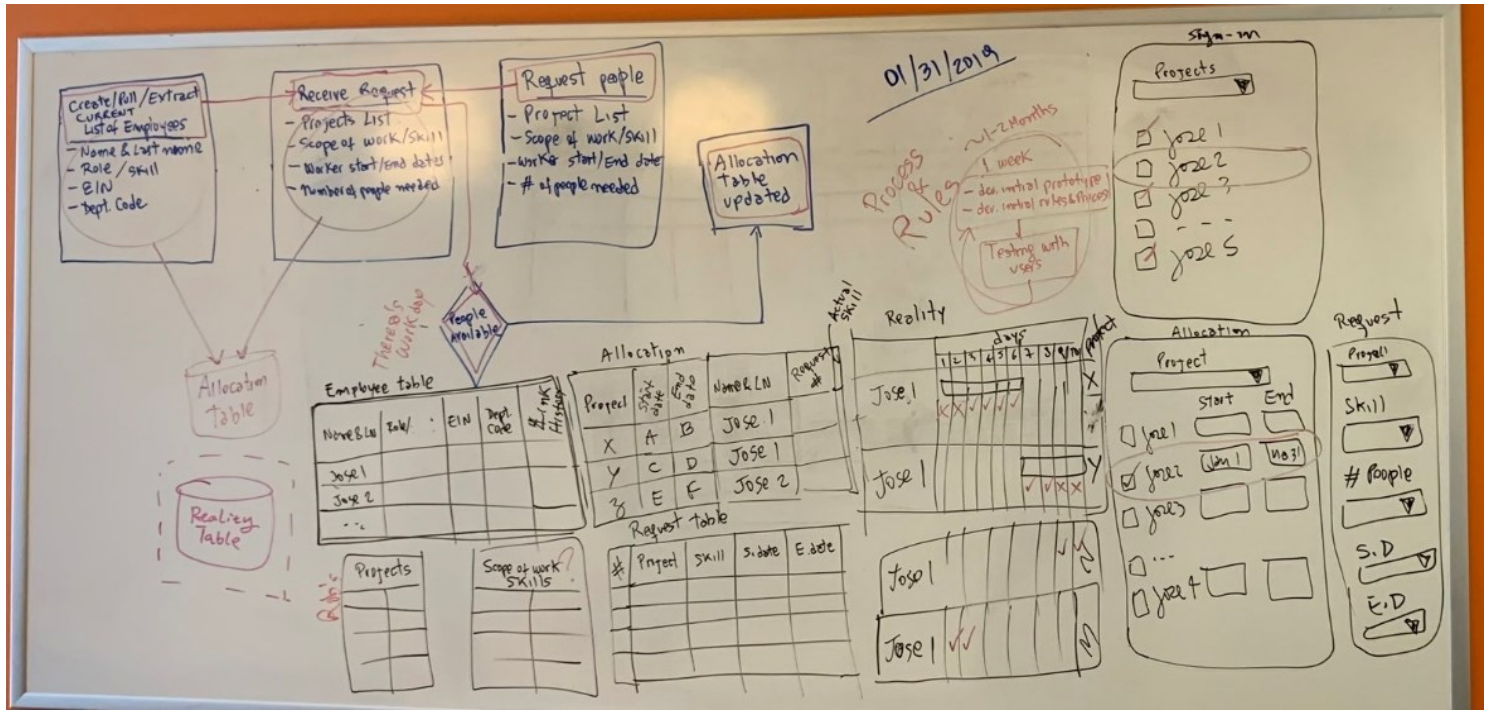

Figure 2: Ideate the ideal resource allocation process and visualizing phone app design ideas on the whiteboard

To be able to iterate fast, the BPI team took advantage of a Low-Code Application Platform and followed concepts of agile development, prototyping only the minimal viable product (MVP) to restrain the scope of each of the sprints. Leveraging the process map and the results of the analysis, the BPI team was able to differentiate features that are critical to fulfil the stakeholders' needs. By phasing out the development, the BPI team created the first prototype within three weeks, and some of the less complicated iterations were released even within one week. Prototyping explores potential solutions intuitively, so the abstract design ideas can be turned into products to inspire further comprehensive solutions. Fast iterations encouraged the stakeholders to work with the BPI team as they feel involved when their feedback was addressed quickly in the next version of the prototype. One example of an iteration was to decide whether the labor group would like to click through a step-by-step wizard rather than filling a request form that needs to scroll down to see more questions. As the volunteers from the labor group tested both designs, the BPI team received first-hand feedback when the users tested the prototype, which is not going to be very different from the final version. The testing process only took an hour with almost half of the future user provided their feedback that the form seems easier to navigate and fill out information. Within one month, the BPI team was able to iterate through 132 prototype versions and fully explored every solution with the stakeholders. 


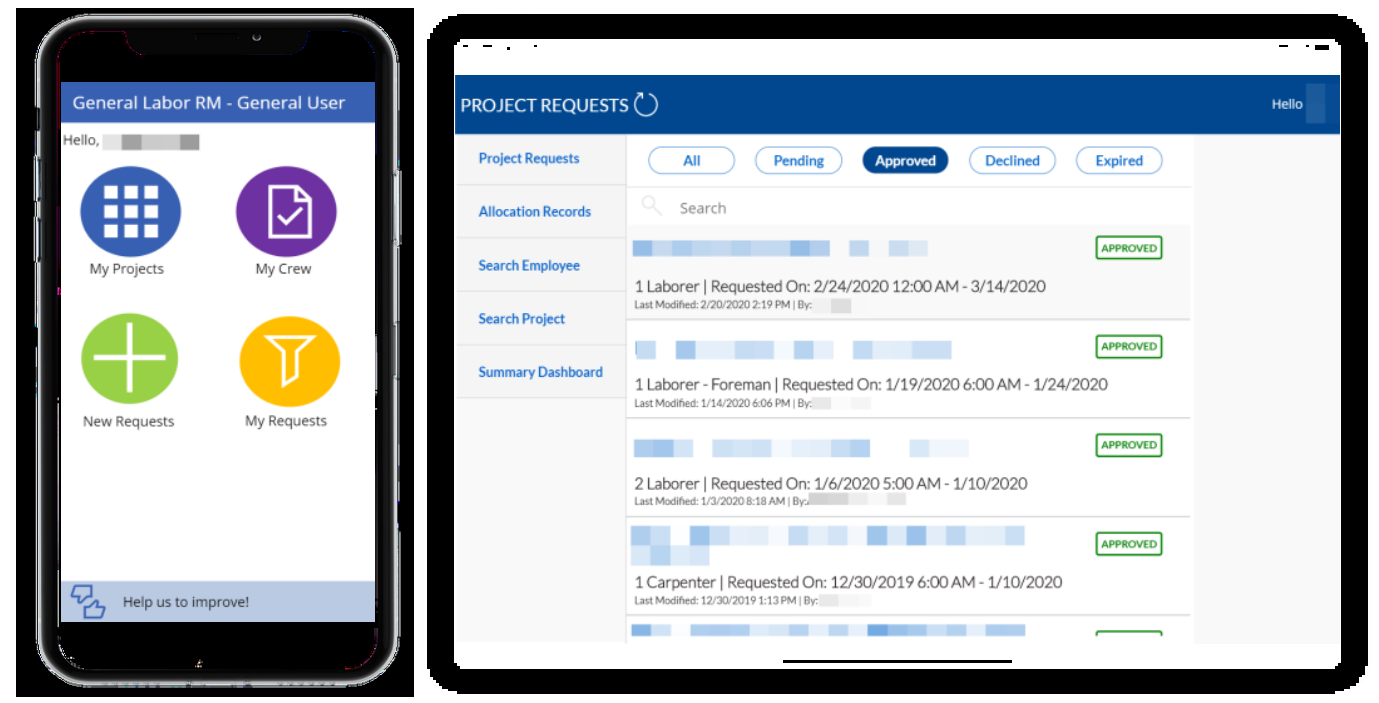

Figure 3: Labor Resource Management application: Field Module (Left) and Manager Module (Right)

\section{Implementation: Company-wide Piloting and Collecting Feedback}

When the design ideas converged and formed a robust solution for this labor management process, the BPI team announced an end to the prototyping phase and moved on to the implementation. The BPI team started the implementation with six months piloting period during which the platform called for volunteers to test it on their projects. Although the BPI team is too small to support the piloting, the labor group was able to identify champions to help with advocating the product and answering users' questions. The champions are also collecting feedback when presenting the application to their fellow workers and communicate the feedback to the BPI team weekly. When the feedback requested new features, the BPI team went through another round of ideation to come up with the most appropriate solution. As the application attracted a substantial amount of the projects and users, the labor group officially started implementing the app within the whole labor group for every project. Till today, all 71 superintendents in the group have downloaded the app on their company phone and are sending in labor requests at least once per week.

\section{DISCUSSIONS}

Visiting the job site and shadowing the users, helped members of the BPI team build empathy and strengthen the perception of the challenges. The project information collected during job walks led to additional contexts to the root cause of certain issues. The interview sessions conducted before the process mapping allowed the BPI team to capture the current condition and to set the baseline for future improvements. By combining the information from different sources, the BPI team members were able to provide rationales for the stakeholders' needs by thinking from their perspective. Hence, this investigation process helped establish a solid foundation built on a wide range of information and knowledge collected in the early phase.

Visualization is important in this phase. The sensation and the emotion built previously by observing and shadowing the stakeholders is hard to be converted into 
accurate verbal communication. By thinking like a designer, sensation and emotion can be expressed by drawings, sketches, and maps. Process mapping on the whiteboard, for instance, provides another way to communicate and form agreements quickly within a team and with the stakeholders. As discovered by Nigel Cross in his research, designers need to interact with an external representation to think in multiple perspectives about future possibilities (Cross, 2018). Creating visualizations expanded the problem space of the task, enabling the BPI team to step back and look at the problem from different perspectives.

Design Thinking emphasizes a deep understanding of the users' needs and a humandriven process. The BPI team recognized that involving the users in the development process by incorporating their feedback through fast iterations significantly increased the users' confidence in the successful implementation of the application. As the team phase out the whole development into smaller sprints, the users can interact with a new version of the prototype every few days and are able to provide relevant feedback. Another key point that enabled the BPI team to involve the users in the prototyping phase actively is the emergence of the Low-Code Application Platform (LCAP) that supports rapid application development, one-step deployment, execution, and management using declarative, high-level programming abstractions (Vincent et al., 2019). By developing in a LCAP environment, the BPI team minimized the development turn-around time since the platform allows business analysts to build and test applications that are close to the quality of the final product without the traditional software development support. Since the feedback is addressed within a few weeks, the users are motivated to provide more suggestions that can add value in building a sustainable solution. This human-centered process also builds the foundation for the next step of implementation.

Implementing a new solution that involves behavior change has always been a challenge for most companies (Armstrong and Gilge, 2016). Since the application was developed with the users' involvement, the stakeholder group can identify champions to help advocate the product and answer questions. Also, since the application was branched from the current process, the volunteers soon found it value-adding to their work. Most of the volunteers did not need a sophisticated training session to start working on the app. The volunteer system reduced the effort to identify potential users, and the feedback mechanism empowered participants to directly raise improvement suggestions and receive responses as the new features were released. Therefore, this forms a positive loop that keeps the initial users engaged. A case study of 117 people from three large Australian construction organizations reveals that one of the most powerful tools for innovation diffusion is word-of-mouth wisdom from committed individuals who mentor and support each other (Peansupap and Walker, 2005). As the initial users introduced the new tool to their co-workers, the labor resource management application started to spread within the labor group. Before the labor group announced the mandate of the app for requesting the labor resource, $42 \%(30 / 71)$ of the superintendents had started to use this application to communicate their labor request to the managers. As the new solution formed a solid user group and pockets of excellence, natural transitioning became possible and significantly reduced the cost of change compared to a hard transition.

Through the case study, the idea of Design Thinking resembles the pull versus push implementation approach under the discourse of Lean management. In both practices, the solutions are solicited completely in collaboration with the stakeholders to build a sustainable solution customized to improve the current condition (i.e., adding value). In a Lean organization, the employees are empowered to self-identified and self-initiated 
process improvements considering that they will make the most valuable decisions (Maestas and Parrish, 2014). The Design Thinking approach is a perfect fit as a problemsolving strategy that promotes a collaborative environment with the stakeholders to drive continuous improvement in a Lean organization.

\section{CONCLUSIONS}

The BPI team at DPR Construction created a solution with the stakeholders that is 1) easy and intuitive to use and 2) can be easily adopted by the stakeholders with minimum training efforts. The case study illustrated how a Design Thinking approach was implemented in developing a mobile-based solution that fulfilled the needs of the stakeholder and achieved a higher adoption rate after implemented.

The paper discusses the philosophy to think from a designer's perspective and depicts its benefits in solving a problem in a way resembling the pull (instead of push) approach. In particular, it emphasizes that a thorough understanding of users' needs can contribute to a greater value creation in designing solutions and managing projects. Hence, it suggests that the Design Thinking approach can be applied not only in managing deliveries of construction projects (Fischer et al., 2017) but also regional or companylevel process improvements or application development.

The paper also discovered that the emergence of Low-Code Application Platform that enables citizen developers to quickly develop applications through a user-friendly graphical user interface (GUI) and application program interfaces (APIs) has great potential in developing a successful business application since the platform significantly reduced the development turn-around time; thus the organization can move faster in finding appropriate solutions that meet the demands of the employees. However, even though the cost for creating a new business application is significantly reduced by the LCAP, the cost for governance and corporate support for maintaining the applications and the platform could post new challenges for a company.

One limitation of this paper is that there was not enough time and information for the BPI team to test the solution's sustainability since the labor group started implementing the new software. The labor group has already created a wish list for new features. Evaluating and prioritizing the requests and phasing out the new development could be the next challenge. For the next step, the authors foresee the BPI team could look into the information collected from the application to provide the leaders of the labor group with critical operation insights such as labor hour utilization and capacity deficiency to enhance the labor allocation.

\section{REFERENCES}

Acar, E., Kocak, I., Sey, Y. and Arditi, D. 2-05. "Use of information and communication technologies by small and medium-sized enterprises (SMEs) in building construction." Construction Management and Economics, 23(10), 713-722.

Armstrong, G. and Gilge, C. 2016. KPMG Global Construction Survey: Building a Technology Advantage. KPMG International, San Francisco.

Cross, N. 2018. Design thinking: understanding how designers think and work. Bloomsbury Visual Arts, London.

DPR 2020. DPR Construction, <https://www.dpr.com/> (May 23, 2020).

Fischer, M., Khanzode, A., Reed, D. and Ashcraft, H. W. 2017. Integrated Project Delivery. John Wiley \& Sons, Incorporated, Somerset. 
Gambatese, J.A., and Hallowell, M. 2011. "Enabling and measuring innovation in the construction industry." Construction Management and Economics, 29(6), 553-567.

Johansson-Sköldberg, U., Woodilla, J. and Çetinkaya, M. 2013. "Design Thinking: Past, Present and Possible Futures." Creativity and Innovation Management, 22(2), 121146.

Maestas, A., and Parrish, K. 2014. "Exploring the Roots of Lean Culture at DPR Construction: A case study in lean culture." Understanding and improving project based production: 22nd Annual Conference of the International Group for Lean Construction, Oslo, Norway 25-27 June, 1413-1424.

Peansupap, V. and Walker, D. 2005. "Factors Affecting ICT Diffusion: A Case Study of Three Large Australian Construction Contractors." Engineering, Construction and Architectural Management, 12(1), 21-37.

Renz, A. and Solas, M. Z. 2016. Shaping the Future of Construction: A Breakthrough in Mindset and Technology.World Economic Forum, Geneva.

Sargent, K., Hyland, P., and Sawang, S. 2012. "Factors influencing the adoption of information technology in a construction business." Construction Economics and Building, 12(2), 72-86.

Schottle, A.D., Maria, L., and Mingle, B. 2019. "From Concept Development to Implementation: Choosing by Advantages Across an Organization." Proc. 27th Annual Conference of the International Group for Lean Construction, Dublin, Ireland, 987-998.

Spitler, L. and Talbot, L. 2017. "Design Thinking as a Method of Improving Communication Efficacy." 25th Annual Conference of the International Group for Lean Construction, Heraklion, Greece, 437-444.

Tschimmel, K. 2012. "Design Thinking as an effective Toolkit for Innovation." Proc. XXIII ISPIM Conference: Action for Innovation: Innovating from Experience, International Society for Professional Innovation Management, Barcelona, 1-20.

Vincent, P., Iijima, K., Driver, M., Wong, J., and Natis, Y. 2019. "Magic Quadrant for Enterprise Low-Code Application Platforms." Gartner Publications, Gartner, $<$ https://www.gartner.com/doc/reprints?id=1-1OC4M37Y> (May 28, 2020). 